Espacio, Tiempo y Forma, Serie II, Historia Antigua, t. 23, 2010, págs. 341-368

\title{
Oficiales astures en las legiones romanas
}

\author{
Asturian officers in the roman legions
}

Narciso Santos Yanguas*

\begin{abstract}
RESUMEN
ABSTRACT

Los astures tomaron parte, aunque en número reducido, como oficiales y suboficiales en las legiones romanas que contribuyeron a la conquista, administración y romanización de las provincias del Imperio.

Junto a ellos otros militares, aunque su procedencia astur no este claramente atestiguada, estuvieron vinculados en el Norte peninsular a la legión VII Gemina.

En todos los casos estos soldados legionarios condicionarían su vida al suelo de los astures tanto durante los años de su servicio militar como después de su liocenciamiento al establecerse en el mismo.

\section{PALABRAS CLAVE:}

Ejército romano, soldados astures, legiones, oficiales y suboficiales, conquista, administración, romanización, establecimiento en suelo astur.

Asturians can be found, though in reduced numbers, as officers and subofficers attached to the Roman legions that undertook the conquest, administration and romanization of imperial provinces.

There were other soldiers as well, though their Asturian origin are not clearly attested, attached to the VII Legion (Gemina) in the Peninsular North.

In practically all cases, these soldiers accommodated themselves to Asturian life both during their military life and after they were licensed, since many settled down in the territory.

\section{KEYWORDS:}

Roman army, asturian soldiers, legions, officers and subofficers, annexation, administration, romanization, settlement in asturian territory.
\end{abstract}

\footnotetext{
•Facultad de Letras. Universidad de Oviedo. C/ Alfonso Martínez s.n. 33011.nsantos@uniovi.es.
} 


\section{INTRODUCCIÓN}

No pretendemos realizar en las páginas siguientes un mero estudio prosopográfico de los soldados de origen astur que, como oficiales o suboficiales, tomarían parte en el marco de los diferentes cuerpos legionarios del ejército romano.

Realmente nuestro propósito va mucho más allá: trataremos de analizar el proceso de integración de tales militares en el contexto de las tropas que contribuyeron a la conquista de los diferentes territorios, a su control y aprovechamiento económico, así como a la organización administrativa de los mismos y a la romanización de las poblaciones con las que entran en contacto.

De esta manera, tras haber analizado y descrito monográficamente ya en otros trabajos anteriores ${ }^{1}$ la configuración e historia de cada una de las unidades militares que portan el étnico Asturum en su denominación, vamos a tratar de catalogar a continuación a los individuos procedentes del conventus jurídico de los astures que estuvieron enrolados, de uno u otro modo, en el marco de las tropas legionarias romanas en la escala militar de oficiales o suboficiales.

Su número no será excesivamente amplio, aunque en ocasiones hagamos mención de algunos soldados cuya procedencia, a pesar de no ser de origen astur propiamente dicho (o, más bien, de no aparecer confirmada expresamente en ningún documento), se relaciona con territorios hispanos vinculados directamente al Norte peninsular, hasta el extremo de que gran parte de su existencia transcurriría en suelo astur, no solo durante el desarrollo de su actividad militar sino también en los años posteriores a su licenciamiento².

Por lo que se refiere al origen de dichos militares un elemento referencial de primer orden lo podría constituir el lugar de hallazgo de los documentos (en casi todos los casos inscripciones de carácter funerario).

A este respecto es posible que algunos de tales enclaves haya que identificarlos con ciertos asentamientos astur-romanos de la Antigüedad, aunque en la mayoría de las ocasiones desconocemos tanto su toponimia concreta como la importancia y significado históricos que alcanzarían a lo largo de su historia más remota.

\section{MILITARES ASTURES EN LAS LEGIONES}

Dadas las especiales características de organización (administrativa y social) de gran parte de las poblaciones astures en el momento a que nos estamos refiriendo (básicamente el siglo I de nuestra era y las décadas correspondientes a la

\footnotetext{
1 Ver, por ejemplo, el análisis llevado a cabo en SANTOS, N.: Ejército romano, administración y vida civil en territorio de los astures, Astorga, 2006.

2 El apéndice final nos permitirá hacernos una idea aproximada de ello.
} 
centuria siguiente) el número de individuos astures que, por su status jurídico podía integrarse en las tropas legionarias sería muy reducido, al menos hasta los primeros momentos del siglo II.

Será entonces cuando, como consecuencia paulatina de la aplicación del ius Latii en todas sus vertientes (individual a las personas y colectiva a los municipios), el número de personas con estatuto privilegiado aumentaría de manera considerable $^{3}$

De la misma manera se añadirían a este colectivo los hijos de antiguos militares enrolados en las tropas auxiliares, cuyos descendientes recibirían, desde tiempos de Claudio, al mismo tiempo que sus padres se licenciaban de las diferentes unidades militares ${ }^{4}$, el derecho de ciudadanía latina junto con otra serie de privilegios $^{5}$.

Como consecuencia de ello su participación en las unidades legionarias se convertiría en un hecho cada vez más frecuente en el ámbito militar romano, resultado sin duda de las nuevas circunstancias políticas y sociales.

A este respecto es posible que, en tiempos de Galba (año 68 d.C.), debido a las urgencias del momento (crisis política en el marco del Imperio romano a la muerte de Nerón), la creación de la legión VII por parte de dicho emperador proclamado por sus tropas (cuerpo militar conocido sucesivamente en un primer momento con los calificativos de Galbiana e Hispana y posteriormente con el de Gemina $^{6}$ ) permitiera un reclutamiento militar que infringía la condición de ciudadano con el objetivo de poder tomar parte en dicha unidad legionaria.

Esta dificultad administrativa sería subsanada a través de la concesión de una ciudadanía de carácter ficticio a cuantos serían enrolados en dicho cuerpo de tropas, entre quienes posiblemente se hallarían algunos individuos de origen astur.

Ahora bien, si la nueva dinastía inaugurada por Vespasiano (los Flavios) favorecía la presencia de individuos astures en el marco legionario romano, este mismo hecho se vería frenado por el cierre del segundo proceso de desmilitarización del territorio peninsular ibérico, a consecuencia del cual únicamente permanecería acampada (además en el Norte peninsular) la legión VII Gemina7.

3 Plinio el Viejo, Historia natura/ 3.30. Para ampliar estos aspectos remitimos, entre otros, a WIEGELS, R.: «Das Datum der Verleihung des ius Latii and die Hispanier: zur Personal- und Municipalpolitik in den ersten Regierungsjahren Vespasians", Hermes, 106 (1978) pp. 196 ss.

4 PEREA, S.: «Los diplomas militares: documentos singulares para la integración jurídica y social de los soldados peregrini al servicio de Roma. Una introducción a su estudio», Formas de integración en el mundo romano, Madrid, 2009, pp. 97 ss

5 SANTOS, N.: «Ciudadanos y extranjeros en el ejército romano: el caso de los astures», Ciudadanos y extranjeros en el mundo antiguo: segregación e integración, Madrid, 2007, pp. 175 ss.

6 PALAO, J.J.: Legio VII Gemina (pia) felix. Estudio de una legión romana, Salamanca, 2006, pp. 43 ss.

7 SANTOS, N.: «La crisis del año 68-69 y la reorganización definitiva del ejército romano de ocupación en territorio de los astures», Aquila legiones, 7 (2006) pp. 109 ss. 
Sin embargo, a dicha unidad militar hemos de añadir un grupo de cuerpos de tropas auxiliares, que dependerían de ella: durante el período de reorganización definitiva del ejército romano de ocupación en territorio hispano (años 70 a 98) el ala II Flavia, la cohorte I gálica, la cohorte II gálica, la cohorte I de los celtíberos y la cohorte lucense, mientras que durante el período de tranquilidad que supone el siglo II (en realidad desde el año 98 al 193) el ala II Flavia, la cohorte I de los celtíberos y la cohorte I gálica ${ }^{8}$.

Todas estas circunstancias condicionarían de forma sustancial el hecho de que no solo la cantidad de oficiales y suboficiales legionarios procedentes del conventus Asturum fuera escasa sino que, incluso, en el caso de los soldados sin graduación, tampoco la aportación del suelo de los astures constituya hasta la fecha una cifra significativa ${ }^{9}$.

Hemos de partir de la situación de que el asentamiento estable de unidades de tropas legionarias como ejército de ocupación en territorio de los astures en los años inmediatos a la finalización de las guerras astur-cántabras traería consigo el hecho de que algunos individuos de la zona (seguramente los menos) fueran reclutados como soldados en el marco de dichos cuerpos militares ${ }^{10}$.

Por ello es posible afirmar que, en una primera fase, más que de participación directa de elementos astures en las legiones romanas, tengamos que referirnos, al menos hasta el momento de creación de la legión VII por Galba (10 de junio del año 68), a soldados legionarios llegados de regiones exteriores a la Península lbérica en su gran mayoría ${ }^{11}$.

Sin embargo, una vez cumplido su servicio militar (o incluso mientras se hallaban en activo) acabarían echando raíces en suelo astur, donde encontrarían familia y, en casos relativamente abundantes, se asentarían inmediatamente después de su licenciamiento.

Debido a ello se distinguirían claramente dos fases en cuanto a la presencia de elementos astures en los cuerpos legionarios romanos (tanto en el caso de oficiales-suboficiales como en el de soldados sin graduación):

A. La primera tendría lugar entre el momento del cierre de las campañas militares en el Norte peninsular y la configuración y establecimiento de la legión VII Gemina en el emplazamiento campamental de León, sustituyendo en el mismo a la

8 ROLDÁN, J.M.: «El ejército hispánico de Vespasiano a Diocleciano: una institución de la España romana imperial», Estudios en homenaje a D.Claudio Sánchez Albornoz en sus noventa años, Buenos Aires, 1983, pp. 563 ss. Cf. SANTOS, N.: «Presencia militar romana en el Norte peninsular en tiempos de los Antoninos: un ejército para la paz», Lancia, 7 (2009) 9 ss.

9 De cualquier forma creemos conveniente separar para su análisis ambos grupos. Cf. SANTOS, N.: «Soldados legionarios de origen astur en el ejército romano», Studia Zamorensia (en prensa).

10 En este sentido hemos de tener en cuenta que el reclutamiento tardorrepublicano en Hispania no solo se llevaría a cabo entre emigrantes itálicos sino que pasarían a formar parte de las unidades legionarias indivuos hispanos que contaban con el derecho de latinidad.

11 SANTOS, N.: «La consolidación del ejército romano de ocupación en territorio de los astures», Sautuola, 12 (2007) pp. 112 ss. 
legión VI Victrix con unos años de intervalo entre el abandono del campamento por ésta y la ocupación (o reocupación) del mismo por parte de aquélla ${ }^{12}$.

Durante esas décadas, que se inician con el primer proceso de desmilitarización operado en el marco del ejército romano de conquista en el Norte peninsular, a la finalización de los enfrentamientos bélicos, quedarían en el territorio septentrional hispano solamente 3 legiones, dos de ellas acampadas en territorio de los astures, la VI Victrix y la X Gemina ${ }^{13}$.

Por ello no resulta extraño que, durante este período, los soldados legionarios de los que tenemos noticias (muchas veces exclusivamente a través de sus epitafios) pertenezcan a dichos cuerpos de tropas, o bien a la legión II Adiutrix, al parecer vinculada temporalmente al arco nordoccidental hispano en el transcurso de la crisis de los años 68-69.

Con el abandono del territorio hispano por parte de ambas legiones (VI Victrix y X Gemina) se cierra el segundo proceso de desmilitarización de las regiones septentrionales ibéricas, proceso que había iniciado ya hacia el año 40 la IIII Macedónica al desplazarse desde su campamento en territorio de los cántabros ${ }^{14}$.

B. La segunda época se corresponde con el acuartelamiento de la legión VII Gemina en el Norte peninsular y el desarrollo de su historia en los siglos siguientes, por lo que no es de extrañar que desde ese momento (en torno al año 74 como fecha definitiva de asentamiento continuado en León, y hasta el siglo IV) sean únicamente militares de dicho cuerpo los que se documenten en territorio de los astures, algunos de ellos de origen astur y otros, tras la realización de su servicio militar, radicados familiar y socialmente en dicho suelo.

Este hecho se vería favorecido, además, por las disposiciones del emperador Vespasiano (inmediatamente después de la promulgación del ius Latii para toda Hispania ${ }^{15}$ ) tendentes a reclutar a los individuos de las tropas legionarias (y auxiliares) en el espacio geográfico próximo a los lugares de acuartelamiento de los mismos $^{16}$

Junto a ello no podemos olvidar la colaboración existente entre algunos destacamentos (vexillationes) de la legión VII Gemina (y otras unidades de tropas auxiliares dependientes de ella ${ }^{17}$ ) y los miembros de la administración imperial (procuratores metallorum y beneficiarii, es decir subalternos a las órdenes de los

12 GARCÍA MARCOS, V.: «Novedades acerca de los campamentos romanos de León», Arqueología militar romana en Hispania, Madrid, 2003, pp. 167 ss.

13 Estrabón, Geografía 3.4.20.

14 PÉREZ, C.: «Asentamientos militares en Herrera de Pisuerga», Los finisterres atlánticos en la Antigüedad, Madrid, 1996, pp. 91 ss.

15 Plinio el Viejo, Historia Natural 3.30. Cf. BOSWORTH, A.B.: «Vespasian and the Provinces. Some Problems of the Early 70 s», Athenaeum, 51 (1973) pp. 49 ss.

${ }_{16}$ Ver, entre otros, WATSON, G.R.: "Conscription and Volunteering in the Roman Imperial Army», SCl, 1 (1974) pp. 90 ss., y «Conscription and Voluntary Enlistment in the Roman Army», PACA, 16 (1982) pp.46ss.

17 Como la cohorte I de los celtíberos, la cohorte I gálica o el ala II Flavia. 
libertos imperiales encargados del control, explotación y salida hacia Roma de los productos mineros del Noroeste peninsular, especialmente los auríferos) ${ }^{18}$.

Esta unidad de acción conjunta, de carácter administrativo, económico y militar a un mismo tiempo, conlleva que, en muchas ocasiones, hallemos en territorio astur soldados de la legión VII Gemina junto con otros correspondientes a unidades de tropas auxiliares dependientes de ella embarcados en el proceso de producción de los distritos mineros de oro (como mano de obra especializada en muchos casos), así como en su control para el desplazamiento posterior de lo obtenido en dirección a la capital del Imperio.

\section{OFICIALES Y SUBOFICIALES ${ }^{19}$}

A este apartado corresponde la presencia de un número significativo de oficiales, especialmente centuriones, de la única legión que, a partir del año 74, estaría asentada en el Norte peninsular (la VII Gemina) ${ }^{20}$.

Por ello tales militares sobresalen sobre el resto, a pesar de que todavía durante la primera mitad del siglo I d.C. otros cuerpos legionarios quedarían acampados en el Norte peninsular tras el proceso de desmilitarización que siguió a la finalización de las guerras astur-cántabras ${ }^{21}$.

Es por ello que, en esta primera fase, encontraremos a algún oficial o suboficial de probable origen astur enrolado en las legiones VI Victrix y X Gemina, que constituían los dos cuerpos legionarios asentados en territorio de los astures, ya que la IIII Macedónica lo estaría entre la población de los cántabros (abandonando dicho suelo en torno al año 40 del siglo I) 22 .

\section{Lucio Anteyo Flavino ${ }^{23}$}

Militaría en el marco de la legión VII Gemina, donde llegaría a desempeñar el cargo de beneficiario consular, momento en que encontraría la muerte en Estepa (provincia hispanorromana de Bética), lugar en que fue hallado su epitafio ${ }^{24}$ :

18 Para más detalles remitimos a SANTOS, N.: «Una inscripción de Villalís (León): los procuratores metallorum y la administración minera romana del oro en territorio de los astures», Astorica, 23 (2004) pp. 9 ss.

19 De esta lista excluimos expresamente a Gayo Sulpicio Úrsulo, cuya importancia y significado ha sido analizado ya con anterioridad en otro trabajo: SANTOS, N.: «Gayo Sulpicio Úrsulo, primer comandante asturiano de una legión romana», Aquila legionis, 12 (2009) pp. 41 ss.

20 Para una visión de conjunto de los centuriones correspondientes a dicho cuerpo de tropas remitimos a LE ROUX, P.: «Recherches sur les centurions de la legio VII Gemina», MCV, 8 (1972) pp.119ss.

21 SANTOS, N.: «El final de las guerras astur-cántabras y la desmilitarización del ejército romano en territorio de los astures», ETF ( $H^{a}$ Antigua), 17 (2007) pp. 251 ss.

22 RODRÍGUEZ, J.: Historia de las legiones romanas, Madrid, 2001, pp. 169-170.

23 La enumeración de la lista de legionarios que presentaremos a continuación se regirá por un orden alfabético definido por el nomen de los personajes.

24 CIL II. $4144=$ HAEp ํㅜ 1461. 


\section{M \\ L ANTEIO FLAVINO \\ BF COS CIVI ASTVRIC \\ LEG VII G P F \\ 5 AN XLV STIP XXIII \\ ANTEIVS ANTYCHVS LIB \\ PATRONO OPTIMO \\ BENE MERENTI FECIT \\ SECVNDVM VOLVNTA \\ 10 \\ TEM SVAM}

$\mathrm{D}$ (iis) $\mathrm{M}$ (anibus)/ L(ucio) Anteio Flavino/ b(ene)f(iciario) co(n)s(ulari) civi Asturic(ensi)/ leg(ionis) VII G(eminae) p(iae) f(elicis)/ an)norum) XLV stip(endiorum) XXIII/ Anteius Antychus lib(ertus)/ patrono optimo/ bene merenti fecit/ secundum volunta/ tem suam.

«(Consagrado) a los dioses manes. A Lucio Anteyo Flavino, beneficiario consular, ciudadano asturicense, de la legión VII Gemina pía feliz, de 45 años de edad, con 23 de servicio (militar), el liberto Anteyo Antycho lo erigió para el mejor patrono, que bien se lo merecía, según su propia voluntad».

Lucio Anteyo Flavino sería promocionado al cargo de beneficiario consular mientras desempeñaba sus funciones en el cuerpo legionario asentado en suelo hispano ${ }^{25}$.

Generalmente a estos beneficiarios se les encomendaban servicios en el marco de la administración de las provincias, por lo que aparecen junto al gobernador u otros altos cargos de la estructura administrativa romana.

Se trata de un ciudadano romano, cuyo origen asturicense se refleja claramente en su lápida, que, una vez cumplidos los años de su servicio militar (más de 20), se retiraría a la región bética, aunque es posible pensar igualmente que la muerte le pudo sorprender hallándose todavía en activo, formando parte de un destacamento de la legión VII Gemina en dicho territorio ${ }^{26}$.

En cuanto a la cronología de este personaje, ciudadano de Asturica Augusta, parece corresponder ya al siglo II (en torno a mediados de dicha centuria).

\section{2. (Lucio?) Atilio Vero}

Desempeñaría el cargo de centurión de la legión V Macedónica en el transcurso de los años intermedios del siglo I d.C.

25 Se asignaba dicha denominación al soldado promocionado a otro grado de la milicia por parte de un tribuno, pretor o cónsul. Cf. DAVIES, R.W.: «Joining the Roman Army», BJ, 169 (1969) pp. 208 ss.

26 Otros autores (PALAO, J.J.: Legio VII Gemina (pia) felix. Estudio de una legión romana, Salamanca, 2006, página 179) asignan a este personaje una cronología más avanzada (inicios del siglo III). 
Su presencia en el marco de dicha unidad legionaria nos viene testimoniada a través de dos fuentes de información diferentes (una epigráfica y otra literaria).

En el primero de dichos documentos, de carácter epigráfico, se hace alusión a un veterano de su centuria que moriría en los alrededores del campamento y a quien dedicaría la lápida un soldado en activo de la misma ${ }^{27}$ :

$\begin{array}{ll} & \text { C ROSCIVS } \\ & \text { C F ANI CA } \\ & \text { PITO TROADE } \\ & \text { VETERAN LEG V } \\ 5 & \text { MAC MILITAVIT } \\ & \text { ANN XXIV VIXIT } \\ & \text { ANN LV H S E } \\ & \text { C VALERIVS } \\ & \text { PVDENS MILES } \\ & \text { LEG V MAC ) ATI } \\ & \text { LI VERI HERES } \\ & \text { FIDVCIARIVS } \\ & \text { F C }\end{array}$

$\mathrm{C}$ (aius) Roscius/ C(aii) f(ilius) Ani(ense) $\mathrm{Ca} /$ pito Troade/ veteran(us) leg(ionis) V/ Mac(edonicae) militavit/ ann(is) XXIV vixit/ ann(is) LV h(ic) s(itus) e(st)/ C(aius) Valerius/ Pudens miles/ leg(ionis) V Mac(edonicae) c(enturia) Ati/ li(i) Veri heres/ fiduciarius/ f(aciendum) c(uravit).

«Cayo Roscio Capitón, hijo de Cayo, de la tribu Aniense, originario de Troade, veterano de la legión V Macedónica, militó durante 24 años, vivió durante 55, aquí esta enterrado. Cayo Valerio Pudente, soldado de la legión V Macedónica, perteneciente a la centuria de Atilio Vero, heredero fiduciario, procuró erigirlo».

Por su parte, el segundo documento se relaciona con la obra de uno de los más preclaros historiadores del mundo antiguo, Tácito ${ }^{28}$, quien, al referirse a los sucesos acaecidos en la batalla de Cremona del año 69, añade el siguiente testimonio:

Urgebatur maxime septima legio, nuper a Galba conscripta. Occisi ex primorum ordinum centuriones, abrupta quaedam signa; ipsam aquilam Atilius Verus primipili centurio multa cum hostium strage et ad extremum moriens servaverat.

«Se apremiaba sobremanera la legión VII, hacía poco reclutada por Galba. Fueron muertos 6 centuriones de los primeros órdenes y algunos de sus estandartes capturados; la misma águila (enseña) la había conservado el centurión del primer orden Atilio Vero tras haber dado muerte a muchos enemigos y, al final, morir él mismo».

\footnotetext{
27 A.E. 1912, no 188 = Boletín de la Sociedad Arqueológica Búlgara, 3 (1912) página 5, oㅡ 2.
}

28 Ann. 15.6. 
Al parecer la muerte del primipilo (primer centurión) Atilio Vero habría tenido lugar en los alrededores de Cremona, habiendo servido con anterioridad como centurión bajo las enseñas de la legión V Macedónica en Oescus (Mesia inferior), donde dicha unidad militar habría estado acampada hasta el año 62, momento en el que sería desplazada con el objetivo de pasar a formar parte del ejército de Armenia ${ }^{29}$.

(Lucio) Atilio Vero era originario del territorio hispano, de acuerdo con lo que se deduce de una lápida encontrada en Aeso (provincia Tarraconense), en la que se hace mención de su hija Atilia Vera ${ }^{30}$ :

L AEMILIO

PATERNO P P

PRAEF FABR ) LEG VII G

5 ) LEG I M ) LEG VII CL

) LEG XIII G ) COH V

) $\mathrm{COH}$ IIII PR CCC LEG II AV

ET PP TER DONIS DONAT

AB IMP TRAIANO TORQVI

10

BVS ARMILLIS PHALERI

CORONA VALLARI BIS

IN DACIA SEMEL IN PAR

THIA

ATILIA L FIL VERA BE

15 NE DE SE MERITO

L(ucio) Aemilio/ L(ucii) fil(io) Gal(eria)/ Paterno p(rimi)p(ilari)/ praef(ecto( fabr(um) c(enturioni) leg(ionis) VII G(eminae)/ c(enturioni) leg(ionis) I M(inerviae) c(enturioni) leg(ionis) VII Cl(audiae)/ c(enturioni) leg(ionis) XIII G(eminae) c(enturioni) coh(ortis) V [urb(anae)]/ c(enturioni) coh(ortis) IIII pr(aetoriae) ccc(trecenario) leg(ionis) II Au[g(ustae)]/ et p(rimo) p(ilo) ter donis dona(to)/ ab imp(eratore) Traiano torqui/ bus armillis phaleri[s]/ corona vallari bis/ in Dacia semel in Par/ thia/ Atilia L(ucii) fil(ia) Vera be/ ne de se merito.

«A Lucio Aemilio Paterno, hijo de Lucio, primipilar, prefecto de los obreros, centurión de la legión VII Gemina, centurión de la legión I Minervia, centurión de la legión VII Claudia, centurión de la legión XIII, centurión de la cohorte V urbana, centurión de la cohorte IIII pretoriana, mercenario de la legión II Augusta y primipilo, 3 veces condecorado por el emperador Trajano con torques, brazaletes y faleras, 2 veces con una corona de trinchera en dacia así como en Parthia, Atilia Vera, hija de Lucio, por sí misma (lo dedica) con merecimiento».

29 PFLAUM, H.G. : «Les officiers équestres de la légion VII Gemina », Legio VII Gemina, León, 1970, página 357 , no 2 .

${ }_{30} \mathrm{CIL}$ II.4461. Cf. DOBSON, B.: Die Primipilares. Entwicklung und Bedeutung, Laufbahnen und Persönlichekeitern eines römischen Offiziersrangen, Bonn, 1978, página 208, noำ 83 . 
Si tenemos en cuenta su posterior desempeño del centurionado como primipilo en el marco de la legión VII Gemina será posible concluir que se hallaría vinculado directamente al suelo de los astures, a pesar de que no sería originario de dicha región, transcurriendo sus años de servicio en torno a mediados del siglo $\left.\right|^{31}$.

Además, el hecho de que su hija fuese la esposa de un alto funcionario legionario, como nos muestra la inscripción que dedica a su marido, nos hace pensar que la vinculación de su padre con el Norte peninsular pudo haber estado más enraizada de lo que a simple vista puede parecer, incluyendo un hipotético origen astur de su yerno.

\section{Aurelio Frontón}

Este centurión aparece reseñado cumpliendo su servicio militar en la legión VII Gemina de acuerdo con su inscripción funeraria (en la actualidad fragmentada) descubierta en el emplazamiento de la misma ${ }^{32}$ :

$\begin{array}{ll}\text { P AT } \\ & \text { RV } \\ & \text { LEG G F } \\ 5 \quad \text { I AV LI } \\ \text { FRO ON } \\ \text { IS AN XV } \\ \text { AE XVI } \\ \quad \text { C }\end{array}$

[D(iis) M(anibus) s(acrum)]/ P(ublio) At[ilio]/ Ru[fo mil(iti)]/ leg(ionis) [VII] G(eminae) $\mathrm{f}$ (elicis)/ c(enturia) Au[re]li(i)/ Fro[nt]on/ is an[n(orum) [XX]XV/ ae[r(orum)] $\mathrm{XVI} /$ [h(eres) f(aciendum)] c(uravit).

«Consagrado a los dioses manes. A Publio Atilio Rufo, soldado de la legión VII Gemina feliz, perteneciente a la centuria de Aurelio Frontón, de 35 años de edad con 16 de servicio (militar), su heredero procuró erigirlo».

No es seguro que Aurelio Frontón fuese de origen astur, a pesar de lo cual su vinculación con el territorio leonés sería prolongada, transcurriendo gran parte de su vida en dicho suelo ${ }^{33}$.

${ }^{31}$ LE ROUX, P. : «Recherches sur les centurions de la legio VII Gemina», pp. 119-120, n 1. Cf. PALAO, J.J.: Legio VII Gemina (pia) felix. Estudio de una legión romana, pp. 56, 189 y 192.

32 CIL II.5681 = CMLeón página 31 .

33 RABANAL, M.A. y GARCIA, S.: Epigrafía romana de la provincia de León: revisión y actualización, León, 2001, pp. 182-183, no 122. 
Sin embargo, todos los indicios parecen apuntar a que este personaje sería de origen hispano según se desprende de otros epígrafes hallados en el Norte peninsular, cuyos antropónimos se relacionarían con él ${ }^{34}$.

Vamos a ver como ejemplo una de estas inscripciones hallada en Lugo ${ }^{35}$ :

\section{M S \\ AVRELLIAE MANTIAE \\ ANNORVM XVIII \\ AVRELIVS FRONTO \\ 5 \\ FILIAE F C}

D(iis) M(anibus) s(acrum)/ Aurelliae Mantiae/ annorum XVIII/ Aurelius Fronto/ filiae $f($ aciendum) $\mathrm{c}$ (uravit).

«Consagrado a los dioses manes de Aurelia Mantia, de 18 años (de edad). Aurelio Frontón procuró erigirlo para su hija».

En lo que se refiere a su datación, lo más probable es que haya que identificarla con las décadas intermedias del siglo II, especialmente si tenemos en cuenta el apelativo correspondiente a la legión ${ }^{36}$.

Por otro lado, al batallón del que Aurelio Frontón era centurión pertenecía igualmente el soldado legionario sin graduación Publio Atilio Rufo ${ }^{37}$.

\section{Tiberio Claudio Pompeyano}

Este soldado legionario, según todos los indicios de origen astur, aparece militando en la legión VII Gemina como tesserarius (es decir encargado de llevar la contraseña en el marco de su unidad militar) ${ }^{38}$, o, más bien, como tribunus, dado que el estado fragmentado del epígrafe no permite una reconstrucción del campo epigráfico con absoluta claridad ${ }^{39}$ :

$\begin{array}{cl} & \text { GENIO } \\ & \text { VII G F TIB } \\ & \text { CL POM } \\ & \text { PEIANVS TR } \\ 5 & \text { EX IVSSV G V } \\ \text { reverso: } & \text { EX } \\ & \text { VO } \\ & \text { TO }\end{array}$

${ }^{34}$ CIL II.2588 y A.E. 1967, no 232. Cf. GARCÍA Y BELLIDO, A.: «Nuevos documentos militares de la Hispania romana», $A E A, 39$ (1966) página 139, donde encotramos dos homónimos.

${ }_{35}$ CIL II.2588. Un caso similar podemnos observarlo en A.E. 1967, no 232.

36 LE ROUX, P. : «Recherches sur les centurions de la legio VII Gemina», pp. 136-137, nº 34 .

37 PALAO, J.J.: Legio VII Gemina (pia) felix. Estudio de una legión romana, página 198.

38 Así había sido interpretado en algún momento de la investigación.

39 GARCÍA Y BELLIDO, A.: «Novedades sobre la legio VII gemina, pía, félix», Tierras de León, 12 (1970) página 17, noำ 2 . 
Genio [leg(ionis)]/ VII G(eminae) F(elicis) Tib(erius)/ Cl(audius) Pom/ peianus $\operatorname{tr}$ (ibunus)/ ex iussu G(enii) v(ovit)/ ex/ vo/ to.

«Al Genio de la legión VII Gemina. Tiberio Claudio Pompeyano, tribuno, por mandato del Genio lo consagró. De acuerdo con su promesa».

La dedicatoria de este documento de carácter votivo, aparecido en el lugar de asentamiento del campamento de la legión VII Gemina (Legio) esta dirigida al genio de la unidad militar, y, de acuerdo con el documento en que se detalla, sería erigida en el mismo lugar de su acuartelamiento campamental ${ }^{40}$.

En cuanto a la datación correspondiente a este epígrafe, si tenemos en cuenta los rasgos paleográficos del mismo, puede situarse ya en los momentos finales del siglo I o, a lo sumo, en una cronología que nos llevaría hasta las décadas intermedias de la centuria siguiente ${ }^{41}$, por lo que resulta totalmente extraña la precisión cronológica que García y Bellido parece asignarle (entre los años 249 y $251)^{42}$.

\section{Quinto Cornelio Anteros}

La única referencia que poseemos acerca de este personaje se corresponde con una inscripción de carácter votivo que fue sacada de la muralla de León (lugar sin concretar) y en la actualidad desaparecida ${ }^{43}$ :

\section{NIMPHIS \\ SACRVM \\ Q CORNELIVS \\ ANTEROS IM \\ 5 AGINIFER LEG VII G F \\ CVM VEXILATI \\ ONE V S L M}

Nimphis/ sacrum/ Q(uintus) Cornelius/ Anteros im/ aginifer leg(ionis) VII G(eminae) $\mathrm{f}$ (elicis)/ cum vexil(I)ati/ one v(otum) s(olvit) I(ibens) $\mathrm{m}$ (erito).

«Consagrado a las ninfas. Quinto Cornelio Anteros, portaenseñas de la legión VII Gemina feliz, junto con un destacamento (de la misma), cumplió su voto con agrado».

40 CRESPO, S. y ALONSO, A.: Las manifestaciones religiosas del mundo antiguo en Hispania romana: el territorio de Castilla y León. I. Las fuentes epigráficas, Valladolid, 1999, página 112, no 145.

41 RABANAL, M.A. y GARCÍA, S.: Epigrafía romana de la provincia de León: revisión y actualización, pp.95-96, no 28. Cf. PALAO, J.J.: Legio VII Gemina (pia) felix. Estudio de una legión romana, página 228.

42 Nueve estudios sobre la legión VII Gemina y su campamento en León, León, 1968, página 60

43 HAEp nำ 494. 
Quinto Cornelio Anteros militó como imaginifer (portaenseñas) en el marco de una vexillatio (destacamento) de la legión VII Gemina, por lo que sus funciones se desplegarían prioritariamente (no sabemos a lo largo de cuantos años) por el Norte peninsular.

Tal vez haya que situar su lugar de procedencia en el correspondiente al emplazamiento de dicha legión, en el que se ha encontrado precisamente su dedicatoria a las ninfas como cumplimiento de algún voto ya en las décadas intermedias del siglo II ${ }^{44}$.

Por lo que se refiere al cognomen (Anteros), de origen griego, se halla testimoniado igualmente en una lápida funeraria procedente de Santiago de Compostela ${ }^{45}$.

Este hecho no vincula necesariamente el lugar de origen de este personaje con el territorio galaico, sino que, y a pesar de la posible tendencia a la aceptación de nombres griegos en aquella época ${ }^{46}$, dado el lugar de hallazgo de la inscripción votiva por él dedicada, nos inclinamos más bien por una procedencia astur.

\section{JULIO JULIANO}

Desempeñaría las funciones de signifer (portaestandarte) de la legión VII Gemina de acuerdo con sendos documentos epigráficos hallados en Villalís (León), en los que se observa perfectamente la conjunción existente entre el elemento militar y el administrativo (en relación con la explotación económica de los recursos mineros de oro de la región).

La primera de dichas inscripciones, fechada el día 10 de junio del año 163, con motivo de la conmemoración del aniversario de dicha unidad legionaria, nos ofrece el siguiente texto ${ }^{47}$ :

$$
\begin{array}{ll}
\text { I O M } \\
\text { O SALVTE M AVRELI AN } \\
\text { ONINI ET L AVRELI VERI } \\
\text { VSTOR OB NATALE AQ } \\
\text { AE VEXILLATIO LEG VII G } \\
\text { SVB CVRA LICINI PATER } \\
\text { ) LEG EIVSD ET HERMETI } \\
\text { AVGVSTOR LIB PROC ET LV } \\
\text { CRETI PATERNI DEC COH }
\end{array}
$$

\footnotetext{
${ }_{4}$ RABANAL, M.A. y GARCÍA, S.: Epigrafía romana de la provincia de León: revisión y actualización, pp.112-113, no⒌ 54 .

45 IRG I, no 18. Cf. LE ROUX, P. y TRANOY, A. : «Notes d épigraphie romaine de Galice», MCV, 9 (1973) pp.223-224.

46 LE ROUX, P. : L armée romaine et I organisation des provinces ibériques d Auguste à I invasion de 409, París, 1982, página 212.

${ }^{47}$ CIL II.2552 = ILS $9125=$ A.E. 1910, nํㅜ $3=$ CMLeón p.70.
} 


\section{I CELT ET FABI MARCIAN \\ B F PROC AVGVSTOR ET \\ VLI IVLIANI SIGN LEG \\ EIVSD IIII ID IVNIAS \\ LAELIANO ET PASTORE CO}

I(ovi) O(ptimo) M(aximo)/ [pr]o salute M(arci) Aureli(i) An/ [t]onini et L(ucii) Aureli(i) Veri/ [Aug]ustor(um) ob natale(m) aq[ui/ I]ae vexillatio leg(ionis) VII G(eminae) [F(elicis)]/ sub cura Licini(i) Pater[ni]/ c(enturionis) leg(ionis) eiusd(em) et Hermeti[s]/ Augustor(um) lib(erti) proc(uratoris) et Lu/ creti(i) Paterni dec(urionis) coh(ortis)/ I Celt(iberorum) et Fabi(i) Marcian[i]/ b(ene)f(iciarii) proc(uratoris) Augustor(um) et/ [I]uli(i) luliani sign(iferi) leg(ionis)/ eiusd(em). IIII id(us) Iunias/ Laeliano et Pastore co(n)[s(ulibus)].

«A Júpiter Óptimo Máximo. Por la salud de Marco Aurelio Antonino y de Lucio Aurelio Vero, Augustos, en el aniversario del natalicio del águila (enseña militar), (lo erigió) el destacamento de la legión VII Gémina Feliz, bajo el mando de Licinio Paterno, centurión de la misma legión, y de Hermes, procurador (de las minas), liberto imperial (de los Augustos), y de Lucrecio Paterno, decurión de la cohorte I de los celtíberos, y de Fabio Marciano, beneficiario del procurador imperial, y de Julio Juliano, portaenseña de la misma legión. En el día 4 antes de los idus de junio (día 10 de junio), siendo cónsules Leliano y Pastor».

Por su parte el segundo de tales monumentos, que se fecha el día 22 de abril del año 165, nos confirma que nuestro personaje continúa desempeñando el cargo de signifer de dicho cuerpo de tropas legionarias ${ }^{48}$ :

\begin{tabular}{ll}
\hline & \\
\hline \multirow{2}{*}{$5 \quad$ ANTON } \\
VE B N[ \\
APR MIL \\
COH I GAL VR \\
\\
LI AVGVSTOR LIB PR \\
VAL FLAVI ) COH EI \\
M ET VAL VALENTI \\
C AVGVST IV \\
IANI SIGNIF LEG VII F \\
\\
X K MAIAS PVD
\end{tabular}

[I(ovi) O(ptimo) M(aximo)]/ [pro salute M(arci) Aureli(i)]/ Anton[ini et L(ucii) Aureli(i)]/ Ve[ri Augustor(um) o]b n[atale(m)]/ apr[unculor(um)] mil[ites]/ coh(ortis) I Gal[I(icae) sub c]ur[a Zo/ i]li Augustor(um) lib(erti) pr[oc(uratoris)]/ [et] Val(erii) Flavi(i) c(enturionis) coh(ortis) ei[us/ de]m et Val(erii) Valenti[s b(ene)f(iciarii)/

48 CIL II. $2556=$ ILS 9129. 
[pro]c(uratoris) August[or(um) et] lu[li(i)/ lul]iani sign(iferi) leg(ionis) VII [G(eminae)] F(elicis)/ X K(alendas) Maias Pud[ente et Pollione/ co(n)s(ulibus)].

«A Júpiter Óptimo Máximo. Por la salud de Marco Aurelio Antonino y de Lucio Aurelio Vero, Augustos, en el aniversario del natalicio de las enseñas militares (lo erigen) los soldados de la cohorte I Galica bajo el mando de Zoilo, liberto imperial (de los emperadores) procurador (de las minas), y de Valerio Flavio, centurión de la misma cohorte, y de Valerio Valente, beneficiario del procurador imperial, y de Julio Juliano, portaenseña de la legión VII Gemina Feliz. 10 días antes de las calendas de mayo (22 de abril), siendo cónsules Pudente y Polión (año 165)».

Sabemos, por tanto, que Julio Juliano desempeñaría las funciones de signifer en el marco de la legión VII Gemina en los años correspondientes a la década de los 60 del siglo II ${ }^{49}$.

Es posible que fuera originario de alguna de las regiones que integraban el conventus de los astures, aunque, de no haber sido así, transcurriría gran parte de su vida en dicho territorio, convirtiéndose sin duda en astur de adopción ${ }^{50}$.

De cualquier forma resulta evidente que la unidad militar a la que pertenecía (sin duda algún destacamento de la legión VII Gemina) tendría mucho que ver con la salvaguarda y protección de los productos obtenidos en el aprovechamiento económico de los recursos auríferos de la región leonesa (así como con su salida en dirección a la capital del Imperio), que en aquellos momentos se hallarían en su fase más intensa de explotación ${ }^{51}$.

\section{Julio Marco}

Este personaje militaría igualmente en las filas de la legión VII Gemina (en un destacamento de la misma como se recoge expresamente en la inscripción), donde llegaría a desempeñar las funciones de centurión.

Aparece, en compañía del decurión Valerio Victor, en un documento epigráfico (en la actulidad fragmentado en su parte superior) fechado en tiempos del emperador Antonino Pío (entre los años 138 y 161) ${ }^{52}$ :

\section{-AELI HADRIANI \\ ANTON AVG PII VEX LEG VII G F SVB CVR IVL MAR}

49 GÓMEZ MORENO, M.: CMLeón, pp. 69-70.

50 PALAO, J.J.: Legio VII Gemina (pia) felix. Estudio de una legión romana, pp. 168, 177 y 399.

51 RABANAL, M.A. y GARCÍA, S.: Epigrafía romana de la provincia de León: revisión y actualización, pp.127-129, no 64 y pp.130-131, oㅡ 66 .

52 A.E. 1927, ํo 229. 


\section{$5 \quad$ CI ) EIVSD ET VAL VICTORIS DEC OB DIEM NAT AQVILAE V S L M}

[I(ovi) O(ptimo) M(aximo)/ pro salute Titi] Aeli(i) Hadriani/ Anton(ini) Aug(usti) Pii vex(illatio) leg(ionis) VII G(eminae) f(elicis)/ sub cura lul(ii) Mar/ ci c(enturionis) eiusd(em) et Val(erii)/ Victoris dec(urionis) ob/ diem nat(alem) aquilae/ v(otum) s(olvit) I(ibens) m(erito).

«A Júpiter Óptimo Máximo, por la salud de Tito Elio Adriano Antonino Augusto Pío, el destacamento de la legión VII Gemina feliz, bajo la dirección de Julio Marco, centurión de la misma, y de Valerio Victor, decurión, con motivo (de la celebración) del día del nacimiento del águila, cumplió su voto con agrado».

Julio Marco sería centurión de la legión VII Gemina de acuerdo con este documento epigráfico hallado en la localidad leonesa de Luyego de Somoza, en el que se conmemora el origen (nacimiento) de este cuerpo de tropas (vexillatio) que formaba parte de la misma ${ }^{53}$.

La presencia de este militar en dicho suelo obedecería a la conexión existente entre algunos de los integrantes de la legión VII Gemina y los funcionarios de la administración minera romana con vistas a la explotación de los recursos auríferos del Noroeste peninsular.

Sin duda dicho centurión estaba en servicio en el territorio de los astures en el momento de realizarse esta conmemoración de carácter votivo, por lo que, aunque desconocemos realmente su procedencia, es posible deducir que transcurriría buena parte de su vida militar en el mismo ${ }^{54}$.

\section{Licinio Paterno}

Militaría como centurión de la legión VII Gemina en torno al año 163, teniendo además como signifer (portaenseña) de la misma a Julio Marco, mencionado con anterioridad 55 .

En este documento epigráfico, hallado en la localidad leonesa de Villalís, se reseña la presencia de varios oficiales que formarían parte de dicha legión: en primer lugar destaca este centurión (Licinio Paterno), que desempeñaría una gran parte de su actividad militar en el conventus de los astures ${ }^{56}$.

53 GARCÍA Y BELLIDO, A.: «Nuevos documentos militares de Hispania romana», AEA, 34 (1961) pp.139-142, no 1 .

54 LE ROUX, P. : «Recherches sur les centurions de la legio VII Gemina», página 132, n 23.

55 CIL II.2552 = ILS $9125=$ A.E. 1910, ํㅡ $3=$ CMLeón página 70. Ver igualmente el número 5 de oficiales legionarios y PALAO, J.J.: Legio VII Gemina (pia) felix. Estudio de una legión romana, p.198.

56 LE ROUX, P. : «Recherches sur les centurions de la legio VII Gemina», página 134, n 28. 
Formaban parte también de este conjunto de militares y civiles, que contarían con unos mismos objetivos (control de la explotación de los recursos mineros de oro con vistas a su desplazamiento, más o menos inmediato, a la capital del Imperio) tanto el decurión de la cohorte I de los celtíberos Lucrecio Paterno como el signifer Julio Juliano ${ }^{57}$.

Junto a ellos se nos muestra igualmente el representante imperial para el control de la explotación y aprovechamiento de los recursos mineros de oro de la zona, a saber el procurator metallorum Hermes (liberto imperial) como prueba de la conjunción de intereses existente durante la segunda mitad del siglo I entre el mundo militar, administrativo y económico ${ }^{58}$.

Finalmente, y como subordinado del procurador encargado del distrito minero por parte de la administración imperial, se hace mención del beneficiario del procurador Fabio Marciano.

\section{Lucrecio Materno}

Este soldado de la legión VII Gemina llegaría a desempeñar el cargo de imaginifer de acuerdo con lo que se desprende de un nuevo monumento epigráfico hallado en la localidad de Villalís (León) ${ }^{59} \quad$ :

IO M

PRO SALVTE M AVRELI ANTONIN

ET L AVRELI VERI AVGVSTOR

OB NATALE SIGNOR VEXILLATIO

5 COH I CELTIB SVB CVRA ZOILI

AVGVSTOR LIB PROC ET VAL FLAVI

) COH I GALL ET AELI FLAVI BF PROC

AVGVSTOR ET LVCRETI MATER

NI IMAG LEG VII G F ET IVLI SE

10 DVLI TESSERARI C I C POSITA

IDIBVS OCTOBRIBVS IMPE AVRE

LIO VERO III ET QVADRATO COS

I(ovi) O(ptimo) M(aximo)/ pro salute M(arci) Aureli(i) Antonin[i]/ et L(ucii) Aureli(i) Veri Augustorum/ ob natale(m) signor(um) vexillatio/ coh(ortis) I Celtib(erorum) sub cura Zoili/ Augustor(um) lib(erti) proc(uratoris) et Val(erii) Flavi(i)/ c(enturionis) coh(ortis) I Gall(icae) et Aeli(i) Flavi(i) b(ene)f(iciarii) proc(uratoris)/ Augustor(um) et Lucreti(i) Mater/ ni imag(iniferi) leg(ionis) VII G(eminae) F(elicis) et 199.

57 VON DOMASZEWSKI, A. : Die Rangordnung des römischen Heeres, Colonia-Graz, 1967, página

58 SANTOS, N.: «Una inscripción de Villalís (León): los procuratores metallorum y la administración minera romana del oro en territorio de los astures», pp. 9ss.

59 CIL II.2553 = ILS 9127. 
Iuli(i) Se/ duli(i) tesserari(i) c(ohortis) I C(eltiberorum) posita/ idib(us) octobrib(us) impe(ratore) Aure/ lio Vero III et Quadrato co(n)s(ulibus).

«A Júpiter Óptimo Máximo. Por la salud de Marco Aurelio Antonino y de Lucio Aurelio Vero, Augustos, en el aniversario del natalicio de las enseñas militares (lo erige) el destacamento de la cohorte I de los celtíberos bajo el mando de Zoilo, liberto imperial (de los emperadores) procurador (de las minas), y de Valerio Flavio, centurión de la cohorte I Galica, y de Elio Flavio, beneficiario del procurador imperial, y de Lucrecio Materno, portaestandarte de la legión VII Gemina Feliz, y de Julio Sedulio, teserario de la cohorte I de los celtíberos. Erigida en los idus de octubre (15 de octubre), siendo cónsules el emperador Aurelio Vero por tercera vez y Quadrato (año 167)».

Lucrecio Materno se hallaba sin duda en servicio activo en el momento en que se erige este monumento, desempeñando el cargo de imaginifer (encargado de llevar la estatua del emperador que acompañaba a los cuerpos legionarios) de algún destacamento de la legión VII Gemina en el occidente de la provincia leonesa ${ }^{60}$.

Desconocemos, sin embargo, en la actualidad si nuestro personaje procedería o no del conventus de los astures, aunque es posible que transcurriese una gran parte de su vida en dicho territorio ${ }^{61}$.

En este sentido la onomástica correspondiente a Lucrecio Materno se encuentra ampliamente extendida por los territorios galos e hispanos ${ }^{62}$.

Vamos a analizar como ejemplo el correspondiente a Astorga, descubierto en la calle de la Rua, identificado con una inscripción funeraria en forma cuadran-

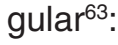

L RVFIO MATERNO

AN XV

L RVFIVS PATER

\section{ET}

5

L(ucio) Rufio Materno/ an(norum) XV/ L(ucius) Rufius pater/ et/ Tryphaena mater.

«A Lucio Rufio Materno, de 15 años de edad, (lo dedican) su padre Lucio Rufio y su madre Tryphaena».

60 GÓMEZ MORENO, M.: CMLeón, página 73.

61 RABANAL, M.A. y GARCÍA, S.: Epigrafía romana de la provincia de León: revisión y actualización, pp. 131-133, no 67 .

62 Para el caso de Hispania ver, por ejemplo, CIL II.231, 378, 1322, 1648...

63 CIL II. 2659. Cf.RABANAL, M.A. y GARCÍA, S.: Epigrafía romana de la provincia de León: revisión y actualización, página 275, $\mathrm{n}$ 을. 
Ahora bien, la mayor frecuencia en este último espacio geográfico ${ }^{64}$, junto a las características en que se llevaría a cabo el reclutamiento de este personaje en aquella época, nos llevan a pensar en un origen hispano, y más concretamente de alguna región del Noroeste peninsular65.

\section{Lucrecio Próculo}

Se hallaría integrado como soldado de la legión VII Gemina, en la que desempeñaría las funciones de guardián de las armas (custos armorum) ${ }^{66}$ :

$\begin{array}{cl} & \text { D M } \\ & \text { LVCRETIO PR } \\ & \text { OCVLO ARM } \\ & \text { ORVM CVS } \\ 5 & \text { AN XXXV ET } \\ & \text { VAL AMM } \\ & \text { VXS AN XXV } \\ & \text { LVCRETIO PRO } \\ & \text { IO EORVM } \\ 10 \quad \text { AN I POSVIT } \\ & \text { SOCER PATER } \\ & \text { AVVS VAL M } \\ & \text { ARCELLINVS }\end{array}$

$\mathrm{D}$ (iis) $\mathrm{M}$ (anibus)/ Lucretio $\mathrm{Pr} /$ oculo arm/orum cus(todi)/ an(norum) XXXV et/ Val(eria) Amm(ae)/ uxs(ori) an(norum) XXV/ Lucretio Pro(culo)/ [fil]io eorum/ an(norum) I[III?] posuit/ socer pater/ avus Val(erius) M/ arcellinus.

«(Consagrado) a los dioses manes. A Lucrecio Próculo, guardián de las armas, de 35 años de edad, y a Valeria Amma, su mujer, de 25 años de edad, y a Lucrecio Próculo, hijo de ambos, de 4 (¿) años de edad, lo erigió Valerio Marcelino, suegro, padre y abuelo».

Esta inscripción, descubierta en la muralla de León, y cuya cronología parece corresponder a la segunda mitad del siglo II, recoge a varios miembros de una misma familia, cuyo motivo de fallecimiento desconocemos, pero cuya lápida mortuoria sería levantada por el padre de la mujer, suegro por tanto del cabeza de familia y abuelo del hijo de los dos personajes adultos ${ }^{67}$.

64 ABASCAL, J.: Los nombres personales en las inscripciones latinas de Hispania, Murcia, 1964, pp.417-420.

65 PALAO, J.J.: Legio VII Gemina (pia) felix. Estudio de una legión romana, página 170.

66 CIL II.2668 = CMLeón página 27.

67 RABANAL, M.A. y GARCIA, S.: Epigrafía romana de la provincia de León: revisión y actualización, pp.249-250, no 199 . 
Valerio Marcelino dedica este monumento funerario a su yerno, a su hija y a su nieto en la localidad de León, por lo que resulta fácil suponer que probablemente este soldado fuese originario de dicho centro de población o de alguno otro de los correspondientes a los astures.

Aunque en la lápida funeraria no se registra su procedencia de población alguna del Noroeste peninsular (al contrario, por ejemplo, del caso de Tito Montanio Frontón, de origen zoela), una serie de elementos, entre los que sobresalen su onomástica68, así como la decoración del documento epigráfico (con figuras zoomorfas que representan a los difuntos), nos permiten pensar en un origen nordoccidental hispano ${ }^{69}$.

Esta decoración animalística vincula dicho monumento con un amplio grupo de estelas indígenas correspondientes al sur del conventus Asturum y al este del bracaraugustano ${ }^{70}$.

Por su parte el cognomen de la esposa (Amma) se relaciona con las regiones septentrionales de la Península Ibérica ${ }^{71}$, lo que nos confirma que este soldado, si no era originario de la zona, se había unido a una familia radicada en el Oeste peninsular (probablemente de origen astur).

Ahora bien, la función de guardián de las armas (custos armorum), es decir encargado del control del depósito de armas situado en el campamento, no parece haber constituido un rango demasiado elevado en la escala militar (suboficial de escaso rango) ${ }^{72}$ : correspondería a los niveles más bajos del grupo de los principales, hallándose en una situación similar a la del tesserarius, en casi todos los casos con escasas posibilidades de ascenso ${ }^{73}$.

De cualquier forma, en el momento de su muerte Lucrecio Próculo apenas habría tenido una carrera militar de corta duración (entre 12 y 15 años de servicio).

\section{Lucio Mantio Hispano}

Tal vez nos hallemos ante un centurión legionario de origen astur (en todo caso procedente del Noroeste de la Península Ibérica) de acuerdo con el epitafio hallado en territorio norteafricano que le dedica su hijo Lucio Mantio Ceciliano ${ }^{74}$

68 SASTRE, I.: Onomástica y relaciones políticas en la epigrafía del conventus Asturum durante el Alto Imperio, Madrid, 2002, pp. 58-60.

69 PALAO, J.J.: Legio VII Gemina (pia) felix. Estudio de una legión romana, págaina 139.

70 SANTOS, N.: El ejército y la romanización de Galicia, Oviedo, 1988, pp. 221-222.

71 UNTERMANN, J.: Elementos de un Atlas antroponímico de la Hispania antigua, Madrid, 1975, pp.53-54, $\mathrm{n}^{\circ} 7$.

72 LE ROUX, P. : L armée romaine et I organisation des provinces ibériques d Auguste à I invasion de 409, página 236.

${ }_{73}$ No resulta extraño por ello que sean numerosos los soldados que, en el momento de su licenciamiento, no hubieran sobrepasado este escalón. Cf. BREEZE, D.J.: «The Organization of the Career Structure of the inmunes and principales of the Roman Army», BJ, 174 (1974) pp.257ss.

${ }^{74}$ LE ROUX, P.: L armée romaine et I organisation des provinces ibériques d Auguste à I invasion de 409, página 236. 


\begin{tabular}{cl} 
& \multicolumn{1}{c}{ D M } \\
& L MANTI L F \\
& GAL HISPANI \\
& ) LEG VII GE \\
5 & MINAE F \\
& ) LEG III AVG \\
& ) I I P PR V A XXXX \\
& L MANTIVS CAE \\
$10 \quad$ CILIANVS F PA \\
& TRI KAR FECIT
\end{tabular}

D(iis) $M$ (anibus)/ L(ucii) Manti(i) L(ucii) f(ilii)/ Gal(eria) Hispani/ c(enturionis) leg(ionis) VII Ge/ minae f(elicis)/ c(enturionis) leg(ionis) III Aug(ustae)/ c(enturionis) I h(astatae) pr(imae) v(ixit) an(nnis) XXXX/ L(ucius) Mantius Cae/ cilianus f(ilius) pa/ tri kar(issimo) fecit.

«(Consagrado) a los dioses manes de Lucio Mantio Hispano, hijo de Lucio, de la tribu Galeria, centurión de la legión VII Gemina feliz, centurión de la legión III Augusta, centurión de la primera compañía de hastados, que vivió 40 años. Lucio Mantio Ceciliano, su hijo, lo erigió a su queridísimo padre».

A través de otro documento epigráfico, igualmente funerario, dedicado en esta ocasión a la esposa de este mismo personaje por parte de dicho hijo y aparecida igualmente en Lambaesis (provincia romana de Numidia), se nos refleja el cursus honorum de dicho militar en lo que se refiere al desempeño del centurionado en la legión III Augusta75:

$\begin{array}{cl} & \text { D M } \\ & \text { IVNIAE CAE } \\ & \text { CILIANAE } \\ & \text { VXORIS } \\ 5 & \text { MANTI HIS } \\ & \text { PANI) LEG III AVG } \\ & \text { VIX ANNIS XX } \\ & \text { L MANTIVS } \\ & \text { CAECILIANVS } \\ 10 \quad & \text { FILIVS MATRI KAR } \\ & \quad \text { FECIT }\end{array}$

D(iis) M(anibus)/ luniae Cae/ cilianae/ uxoris/ Manti(i) His/ pani c(enturionis) leg(iones) III Aug(ustae)/ vix(it) annis XX[XX]/ L(ucius) Mantius/Caecilianus/ filius matri kar(issimae)/ fecit.

75 CIL VIII.2939. Cf. PITILLAS, E.: «Integración y promoción social de las poblaciones indígenas del Noroeste hispánico dentro del esquema organizativo romano: ejército y minería», MHA, 19-20 (19981999) página 240 nota 48. 
«(Consagrado) a los dioses manes de Junia Ceciliana, esposa de Mantio Hispano, centurión de la legión III Augusta, que vivió 40 años. Su hijo Lucio Mantio Ceciliano lo erigió a su queridísima madre».

Todos los indicios apuntan a que este personaje sería de procedencia hispana; es más, este mismo origen explicaría el desempeño de su primera función militar en el marco de la legión VII Gemina ${ }^{76}$.

Este hecho, así como su pertenencia a la tribu Galeria, es los que nos permite pensar que, dada la proximidad del campamento legionario, su relación, incluso de nacimiento, con la zona de los astures sería plena.

Por lo que se refiere a la cronología de su cursus honorum tal vez haya que situarla en la segunda mitad del siglo II, por lo que su reclutamiento tendría lugar en torno a mediados de dicha centuria ${ }^{77}$.

\section{Tito Montanio Frontón}

Un nuevo encargado del fortín de las armas correspondientes a la legión VII Gemina se recoge en un documento epigráfico hallado en la ciudad de León, que constituye la lápida funeraria de dicho personaje ${ }^{78}$ :

\begin{tabular}{cl} 
& \multicolumn{1}{c}{ D M S } \\
& T MONTANIO \\
& FRONTONI AR \\
& CVS CIVI ZOELAE \\
5 & AN LIII STIP XXVI \\
& T MONTANIVS \\
& MATERNVS \\
& PATRONO OPT \\
& CVRATOR FF LL \\
$10 \quad$ & M POSVIT S T T L
\end{tabular}

$\mathrm{D}$ (iis) $\mathrm{M}$ (anibus) s(acrum)/ T(ito) Montanio/ Frontoni ar(morum)/ cus(todi) civi Zoelae/ an(norum) LIII stip(endiorum) XXVI/ T(itus) Montanius/ Maternus/ patrono op(timo)/ curator f(iscorum) I(egionariorum)/ m(onumentum) posuit s(it) t(ibi) t(erra) I(evis).

«Consagrado a los dioses manes. A Tito Montanio Frontón, guardián de las armas, ciudadano zoela, de 53 años de edad con 26 de servicio (militar), Tito Montanio Materno, encargado del fisco legionario, erigió este monumento al mejor patrono. ¡Que la tierra te sea levej».

76 LE ROUX, P. : «Recherches sur les centurions de la legio VII Gemina», página 136.

77 Su mismo nomen parece apuntar en este sentido. Cf. ALBERTOS, M.L. La onomástica personal primitiva de Hispania Tarraconense y Bética, Salamanca 1966, p. 147.

78 CIL II.5684. 
La milicia de Tito Montanio Frontón que aquí aparece reseñada puede fecharse a finales del siglo I o en las primeras décadas de la centuria siguiente ${ }^{79}$.

Este personaje era originario de la población de los zoelas, situada al sureste de Asturica Augusta ${ }^{80}$.

Los zoelas obtendrían sin duda la condición de municipio (civitas) en los momentos finales de la dinastía Flavia como consecuencia de la aplicación y adaptación del ius Latii vespasianeo a los centros de población del Noroeste peninsular ${ }^{81}$.

Por lo que se refiere al cargo desempeñado por su liberto Tito Montanio Materno (curator ff.II.) se hallaba relacionado con el control y administración de todos los documentos militares que afectaban a la organización de un determinado lugar ${ }^{82}$.

Dicha actividad, de control y supervisión, se correspondería con todo lo relacionado con el aprovisionamiento del ejército (transporte y distribución de víveres), así con la reparación de edificios y vías públicas, sin olvidar la vigilancia y dirección de los talleres militares ${ }^{83}$.

A la finalización de los años de su servicio militar (tal vez con algunos de reenganche) Tito Montanio Frontón se establecería como veterano en la ciudad de León, donde su liberto le dedicaría el epitafio.

\title{
13. Marco Titio Rufo (?)
}

Esta inscripción, en la actualidad desaparecida y sobre cuyo lugar de hallazgo existen serias dudas, aunque podemos pensar que se encontraría en León, nos documenta la existencia de un primipilo (primer centurión) de la legión VII Gemina, cuyo nombre podemos reconstruir siguiendo las indicaciones de Hübner ${ }^{84}$ :

\author{
N ET CASTRORVM \\ AC PATRIAE \\ F SABATINA \\ 5 \\ PP LEG VII G SEVER \\ P F EX CORNI \\ AET EE MM VV \\ ET VRBANO COS
}

\footnotetext{
79 MANGAS, J.: Esclavos y libertos en la España romana, Salamanca, 1971, página 414.

80 Plinio el Viejo, Historia Natural 3.3.27-28.

81 Más detalles en GARCÍA, E.: «Una propuesta de identificación epigráfica de los cives Latini», HAnt, 17 (1993) página.345, nำ 28

82 PALAO, J.J.: Legio VII Gemina (pia) felix. Estudio de una legión romana, página 130, aunque es partidario de una cronología mucho más tardía (al menos un siglo después).

${ }^{83}$ RABANAL, M.A.y GARCÍA, S.: Epigrafía romana de la provincia de León: revisión y actualización, pp.259-260, nํㅜㅇ. 209 .

84 CIL II.2664.
} 
[luliae Mamaeae Aug(ustae) matri/ Aug(usti)] n(ostri) et castrorum/ [et senatus] ac patriae/ [M(arcus) Titius M(arci)] f(ilius) Sabatina/ [Rufus] p(rimus) p(ilus) leg(ionis) VII G(eminae) Sever(ianae)/ [Alexandr(ianae)] p(iae) f(elicis) ex corni/ [culario praef(ectuum) pr]aet(orii) em(inentissimorum) v(irorum)/ [Maximo II] et Urbano $\mathrm{co}(\mathrm{n}) \mathrm{s}$ (ulibus).

«A Julia Mamea Augusta, madre de nuestro Augusto (emperador) y del campamento, así como del senado y de la patria, Marco Titio Rufo(¿), hijo de Marco, de la tribu Sabatina, primer centurión de la legión VII Gemina Severiana Alexandriana pía feliz, ex-corniculario de los prefectos del pretorio, hombres eminentísimos, siendo cónsules Máximo por segunda vez y Urbano (año 234)».

Marco Titio Rufo(¿) desarrollaría su carrera militar, en un principio como corniculario (secretario) de los prefectos del pretorio y posteriormente como primer centurión (primipilo) de la legión VII Gemina en torno al año 234, de acuerdo con lo que se desprende de esta inscripción monumental y honorífica dedicada a Julia Mamea, madre del emperador Alejandro Severo ${ }^{85}$.

Este antiguo pretoriano, posiblemente originario de la Península Itálica, se vería favorecido por una rápida promoción en su carrera militar, puesto que sería elevado directamente desde el nivel de secretario de los prefectos del pretorio al grado de primipilo (primer centurión) de la legión VII Gemina ${ }^{86}$.

\title{
14. .... Vettio
}

La presencia de este soldado enrolado al frente de una centuria de la legión VII Gemina nos viene recogida en una inscripción votiva hallada en Ujo (concejo de Mieres, Asturias), en la que Lucio Corona Severo dedica a Júpiter una inscripción en compañía de su mujer Octavia Prócula ${ }^{87}$ :

\author{
I O M \\ LVCIVS \\ CORONA S \\ EVERVS M \\ 5 ILES LEG VII \\ GEMAE ) VE \\ TTI ET OCTA \\ VIA PROCVLA \\ V S L M
}

85 DOBSON, B.: Die Primipilares. Entwicklung und Bedeutung, Laufbahnen und Persönlichekeitern eines römischen Offiziersrangen, pp. 299-200, no 202.

86 VON DOMASZEWSKI, A. : Die Rangordnung des römischen Heeres, pp. 102-103. Cf. PFLAUM, H.G. : «Les officiers équestres de la légion VII Gemina », página 373, n 23.

87 CIL II.5733 = E.E. 2, no 29. 
I(ovi) O(ptimo) M(aximo)/ Lucius/ Corona S/ everus $\mathrm{m}$ / iles leg(ionis) VII/ Gem(in)ae c(enturia) Ve/ tti(i) et Octa/ via Procula/ v(otum) s(olverunt) I(ibentes) $\mathrm{m}$ (erito).

«A Júpiter Óptimo Máximo. Lucio Corona Severo, soldado de la legión VII Gemina, perteneciente a la centuria de Vettio, y Octavia Prócula cumplieron su voto con agrado».

No conocemos nada más acerca de este centurión de nombre Vettio, aunque hemos de destacar el hecho de que dicho gentilicio, que tal vez fuera de origen itálico, aparece con relativa frecuencia en los documentos epigráficos de la Península Ibérica ${ }^{88}$.

Así, en el marco de otras unidades de tropas legionarias, encontramos, por ejemplo, a Tito Vettio Rufo, centurión de la legión XIII Gemina ${ }^{89}$, así como a Cayo Praecelio Augurino Vettio, tribuno de la legión VII90.

La existencia de este soldado debe ser fechada, así como la del dedicante de la inscripción (Lucio Corona Severo), ya en las décadas finales del siglo II, o tal vez en los primeros años de la centuria siguiente ${ }^{91}$.

\section{Jinete de nombre desconocido, escribano}

Este personaje estuvo enrolado entre los soldados de caballería correspondientes a la legión VII Gemina, desempeñando el cargo de escribano (actarius), de acuerdo con una inscripción vinculada geográficamente al campamento ocupado por dicha unidad militar ${ }^{92}$ :

$\begin{array}{ll} & \text { IMP CAESARI } \\ & \text { M AVREL ANTO } \\ & \text { NINO PIO FELI } \\ & \text { CI AVG PARTHIC } \\ 5 & \text { MAX BRIT MAX } \\ & \text { GERMAN MAX } \\ & \text { PONTIF MAX } \\ & \text { TRIB POT XVIIII } \\ & \text { COS IIII IMP III } \\ & \text { P P PROC } \\ & \text { EQVITES IN } \\ & \text { HIS ACTARIVS } \\ & \text { LEG VII GEM }\end{array}$

88 DIEGO SANTOS, F.: Epigrafía romana de Asturias, Oviedo, 1959, pp.38-39, ํo 1.

89 CIL III.4563.

90 CIL V.331. Cf. PALAO, J.J.: Legio VII Gemina (pia) felix. Estudio de una legión romana, pp.190 y 192.

91 LE ROUX, P. : «Recherches sur les centurions de la legio VII Gemina», página 140, n 42.

92 CIL II.2663 = ILS 2335. 
15

ANT P FEL DE

VOTI NVMINI

MAIESTATIQ EIVS

En el lateral: DEDICAT

$\begin{aligned} & \text { VII K OCT } \\ & \text { CATTIO SAB } \\ & \text { INO II ET CO } \\ 5 \quad & \text { ANVLLINO } \\ & \text { COS }\end{aligned}$

Imp(eratori) Caesari/ M(arco) Aurel(io) Anto/ nino Pio Feli/ ci Aug(usto) Parthic(o)/ max(imo) Brit(annico) max(imo)/ German(ico) $\max ($ imo)/ pontif(ici) $\max ($ imo)/ trib(unicia) pot(estate) XVIIII/ co(n)s(uli) IIII imp(eratori) III p(atri) p(atriae) proc(onsuli)/ equites in/ his actarius/ leg(ionis) VII Gem(inae)/ Ant(onianae) p(iae) f(elicis) $\mathrm{de} /$ voti numini/ maiestatiq(ue) eius./ Dedicat(um)/ VII k(alendas) oct(obres)/ Cattio $\mathrm{Sab} /$ ino II et Co[rn(elio)]/ Anullino/ co(n)s(ulibus).

«Al emperador César Marco Aurelio Antonino Pío Feliz Augusto, Pártico máximo, Británico máximo, Germánico máximo, pontífice máximo, en su XVIIII tribunicia potestad, cónsul 4 veces, con 3 salutaciones imperiales, padre de la patria, procónsul, los jinetes, entre ellos el escribano, de la legión VII Gemina Antoniana pía feliz, devotos de su numen y majestad. Dedicado 7 días antes de las calendas de octubre (25 de septiembre), siendo cónsules Cattio Sabino por segunda vez y Cornelio Anullino (año 216)».

La existencia de este jinete, de nombre desconocido, aparece registrada en una dedicación especial al emperador Caracalla, fechada el día 25 de septiembre del año 216, hallada al parecer en Ruiforco de Torío, desde donde se trasladó a León ${ }^{93}$.

Es posible que dicho personaje, cuyo origen y procedencia no conocemos, además de servir en este cuerpo de legionarios jinetes, a la finalización de su servicio militar se estableciese en alguna zona del territorio leonés y, por tanto, de origen astur ${ }^{94}$.

93 LE ROUX, P. : L armée romaine et I organisation des provinces ibériques d Auguste à I invasion de 409, página 346, nํㅜㄴ. 253.

94 RABANAL, M.A. y GARCÍA, S.: Epigrafía romana de la provincia de León: revisión y actualización, pp.142-144, no 75 . 


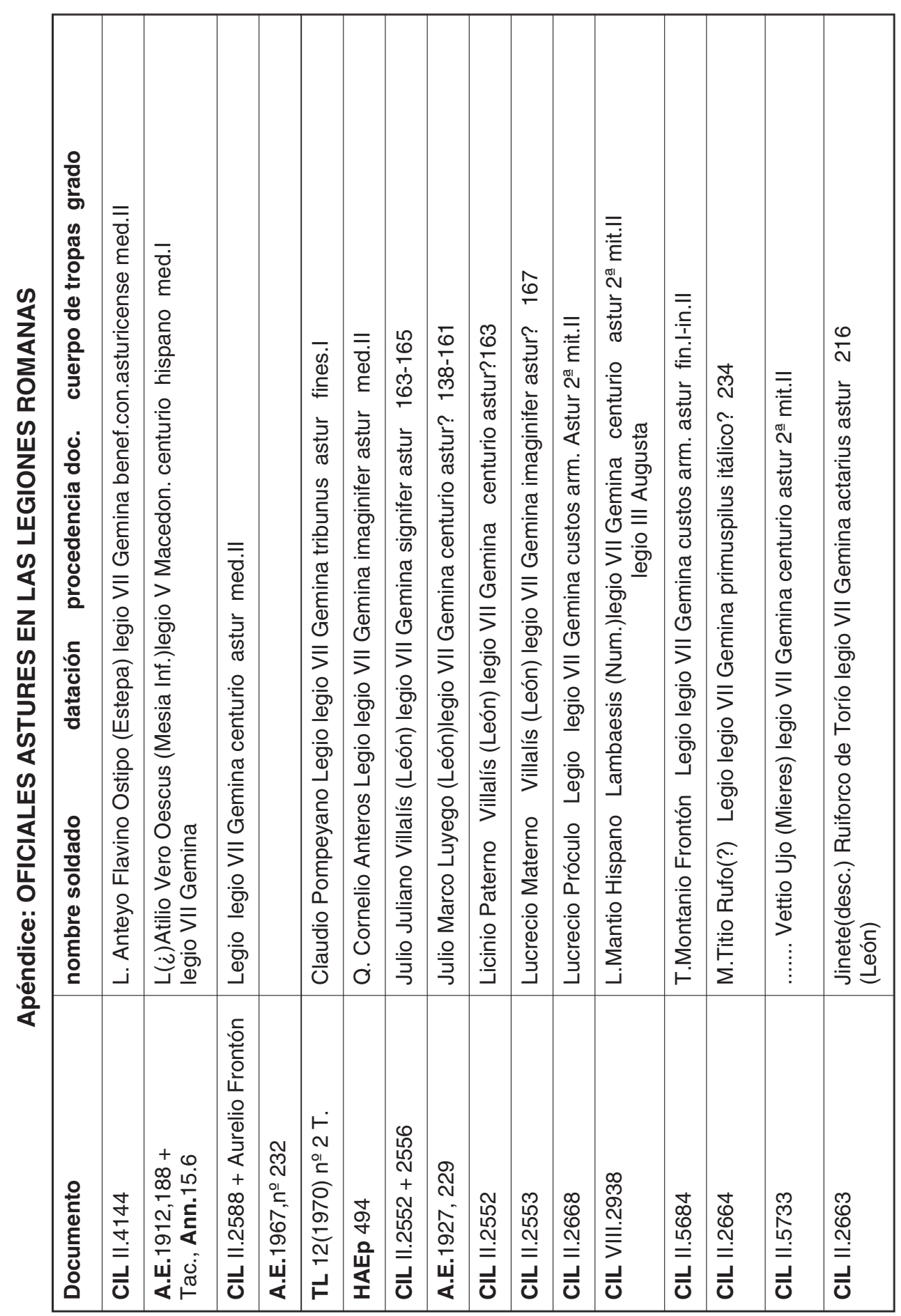


